\title{
Mechanistic Computational Model of Ovarian Steroidogenesis to Predict Biochemical Responses to Endocrine Active Compounds
}

\author{
Michael S. Breen, ${ }^{1}$ Daniel L. Villeneuve, ${ }^{2}$ Miyuki Breen, ${ }^{3}$ Gerald T. Ankley, ${ }^{2}$ and \\ RORY B. CONOLLY ${ }^{1}$ \\ ${ }^{1}$ National Center for Computational Toxicology, Office of Research and Development, U.S. Environmental Protection Agency, \\ 109 T.W. Alexander Drive, Mail B205-01Research Triangle Park, NC 27711, USA; ${ }^{2}$ Mid-Continent Ecology Division, U.S. \\ Environmental Protection Agency, Duluth, MN 55804, USA; and ${ }^{3}$ Biomathematics Program, Department of Statistics, North \\ Carolina State University, Raleigh, NC 27695, USA
}

(Received 16 September 2006; accepted 30 March 2007; published online 13 April 2007)

\begin{abstract}
Sex steroids, which have an important role in a wide range of physiological and pathological processes, are synthesized primarily in the gonads and adrenal glands through a series of enzyme-mediated reactions. The activity of steroidogenic enzymes can be altered by a variety of endocrine active compounds (EAC), some of which are therapeutics and others that are environmental contaminants. A steady-state computational model of the intraovarian metabolic network was developed to predict the synthesis and secretion of testosterone (T) and estradiol (E2), and their responses to EAC. Model predictions were compared to data from an in vitro steroidogenesis assay with ovary explants from a small fish model, the fathead minnow. Model parameters were estimated using an iterative optimization algorithm. Model-predicted concentrations of T and E2 closely correspond to the time-course data from baseline (control) experiments, and dose-response data from experiments with the EAC, fadrozole (FAD). A sensitivity analysis of the model parameters identified specific transport and metabolic processes that most influence the concentrations of $\mathrm{T}$ and $\mathrm{E} 2$, which included uptake of cholesterol into the ovary, secretion of androstenedione (AD) from the ovary, and conversions of AD to T, and AD to estrone (E1). The sensitivity analysis also indicated the E1 pathway as the preferred pathway for E2 synthesis, as compared to the $\mathrm{T}$ pathway. Our study demonstrates the feasibility of using the steroidogenesis model to predict $\mathrm{T}$ and E2 concentrations, in vitro, while reducing model complexity with a steady-state assumption. This capability could be useful for pharmaceutical development and environmental health assessments with EAC.
\end{abstract}

Keywords-Steroid biosynthesis, Mathematical model, Sensitivity analysis, Endocrine disrupting chemicals, Fadrozole, Fish, Cellular metabolism.

Address correspondence to Michael S. Breen, National Center for Computational Toxicology, Office of Research and Development, U.S. Environmental Protection Agency, 109 T.W. Alexander Drive, Mail B205-01Research Triangle Park, NC 27711, USA. Electronic mail: breen.michael@epa.gov

\section{INTRODUCTION}

Steroid hormones play an essential role in a wide range of physiological and pathological processes such as development, growth, reproduction, metabolism, aging, and hormone-sensitive cancers. ${ }^{24}$ Sex steroids are synthesized primarily in the gonads (ovaries and testes) and adrenal tissue and derived from cholesterol through a series of biochemical reactions mediated by multiple cytochrome P450 (CYP) enzymes and hydroxysteroid dehydrogenases (HSD) ${ }^{20,27,36}$ The activity of these steroidogenic enzymes can be altered by various environmental and pharmacologic endocrine active compounds (EAC). ${ }^{10,28,29,32,45,47}$ Our research goal is to better understand the dose-response behaviors of EAC. Our approach is to develop computational systems biology models that describe the biological perturbations at the biochemical level and integrate information towards higher levels of biological organization. This approach will ultimately enable predictions of dose-response behavior at the organismal level. In this paper, we describe a mechanistic model for the biosynthesis of the sex steroids, testosterone (T) and estradiol (E2), and their responses to EAC, which can inhibit the enzymes involved in steroidogenesis.

Data for our computational model were obtained from an in vitro steroidogenesis assay with ovary preparations from a small fish model, the fathead minnow. The fathead minnow was selected as an appropriate animal model for several reasons. ${ }^{2,15}$ This species has a relatively international distribution and is representative of a large and ecologically significant family of fish (Cyprinidae). The fathead minnow is one of the most extensively tested fish species in the world, and published methods exist for its culture, early life-stage, and full life-cycle tests. ${ }^{37,39-41}$ It has a 
relatively rapid life cycle (hatch to sexual maturity in 4-5 months), and timing of reproductive cycle can be effectively controlled using temperature/photoperiod manipulation, ${ }^{38}$ thus enabling a lab to maintain a constant supply of test organisms at a developmental stage suitable for testing. At maturity, females have ovary tissue volumes that are more than sufficient for the in vitro steroidogenesis assay.

A mechanistic steroidogenesis model has several potential applications. First, this type of model helps to obtain an improved understanding of the biochemical responses, and could serve as a basis for the identification of predictive biomarkers indicative of adverse effects. Second, a model that predicts T and E2 responses to EAC can be coupled to multi-organ systems models to include the $\mathrm{T}$ and $\mathrm{E} 2$ regulatory feedback of the hypothalamus-pituitary-gonadal axis. Third, a steroidogenesis model could be used as a basis for predicting the therapeutic and potentially adverse effects of pharmacological and environmental EAC that interact with components of the steroid biosynthesis pathway.

Other models of steroid synthesis have been described in the literature. Selgrade et al. developed a mathematical model to predict plasma levels of E2 during different stages of the menstrual cycle in women from luteinizing hormone ( $\mathrm{LH})$ and follicle stimulating hormone concentrations. ${ }^{34}$ They modeled E2 concentrations as a weighted sum of LH concentration, growth follicle stage, and preovulatory stage. Murphy et al. developed a model for vitellogenesis, a steroidcontrolled process, in female fish. ${ }^{23}$ To model steroidogenesis in the fish, they lumped all reactions occurring between the release of gonadotropin and the production of $\mathrm{T}$ into one Hill equation. Since these models do not include any reactions in the metabolic pathway for steroid synthesis, their ability to predict responses to EACs that inhibit specific steroidogenic enzymes, is limited. Becker et al. developed a probabilistic model of the metabolic pathway for testicular steroidogenesis. ${ }^{5}$ They estimated transition probabilities for the reactions in the pathway, and estimated the maximum steroid secretion rates from ex vivo rat and rabbit testes preparations.

In this study, we describe a model of the intraovarian metabolic and transport processes that mediate steroid synthesis and secretion, and the kinetics for enzyme inhibition by a competitive inhibitor of steroidogenic enzymes, fadrozole (FAD), a model EAC. Below, we first describe the in vitro steroidogenesis experiments with control ovaries and those exposed to FAD, and then the computational model and procedures for parameter optimization and sensitivity analysis.

\section{METHODS}

\section{In Vitro Steroidogenesis Assay with Ovary Explants}

We performed two experimental studies: a baseline (control) study with data sampled at six time points $(2.0,4.0,8.0,14.5,23.0$, and $31.5 \mathrm{~h})$, and a FAD study with control (medium only) and five FAD test concentrations $(2.91,9.7,29.1,97.0$, and $291.0 \mu \mathrm{M})$ with data sampled at $14.5 \mathrm{~h}$. A description of the experiments is provided below.

The fathead minnow in vitro steroidogenesis assay was adapted from the methods of McMaster et al. ${ }^{19,43}$ Briefly, wells of 48 well microplates (Falcon 35-3078, Beckton Dickinson, Franklin Lakes, NJ, USA) were filled with either $500 \mu \mathrm{L}$ of Medium 199 [Sigma M2520, St. Louis, MO, USA] supplemented with $0.1 \mathrm{mM}$ IBMX [3-isobutyl-1-methylxanthene, Sigma I7018] and $1 \mu \mathrm{g} \mathrm{mL}^{-1}$ 25-hydroxycholesterol [Sigma H1015]), in the case of the baseline experiments, and control wells in the FAD experiments, or $500 \mu \mathrm{L}$ of FAD-treated supplemented medium. Dilutions of an aqueous FAD (generously provided by Novartis, Inc., Summit, NJ, USA) stock solution were prepared directly in supplemented culture medium in order to generate a series of five test concentrations of FAD $(2.91,9.7,29.1,97$, and $291 \mu \mathrm{M})$, plus a control for the experiment. After preparing the test plates, ovarian tissue from female fathead minnows (5-6 months old) was added. Baseline experiments had four replicates per time sample, and FAD experiments had six replicates per treatment. Once ovary tissue had been added to all wells, the test plate was removed from the ice and incubated at $25^{\circ} \mathrm{C}$. For the time-course baseline experiments (medium only), the incubation time was $2.0,4.0,8.0,14.5,23.0$, and $31.5 \mathrm{~h}$, with medium and tissue being removed from one set of replicate wells at each time point and immediately replaced with $500 \mu \mathrm{L}$ of $25{ }^{\circ} \mathrm{C}$ water. For the FAD exposure experiments, the incubation time was $14.5 \mathrm{~h}$. At the end of the incubation period, medium from each well was transferred to a microcentrifuge tube and frozen at $-80{ }^{\circ} \mathrm{C}$. Medium samples were stored frozen at $-20{ }^{\circ} \mathrm{C}$ until extracted and analyzed. After removal of the medium, the tissue in each well was removed with forceps and wet weight was measured. T and E2 concentrations in the frozen medium samples were measured by radioimmunoassay (RIA) using an adaptation of methods previously described for fathead minnow plasma samples. ${ }^{4,42}$

For statistical analysis of the steroid data, a Kolmogorov-Smirnov test was used to test data for normality. Bartlett's test was used to test homogeneity of variance. A non-parametric Kruskall-Wallis test was used to test for differences across all treatments, 
and Dunn's test was used to determine which treatments differed significantly from the control. All statistical analyses were conducted using SAS 9.0 (SAS Institute, Cary, NC, USA), except Dunn's test which was conducted using GraphPad Instat 3.01 (GraphPad Software, San Diego, CA, USA). Differences were considered significant at $p \leq 0.05$.

To compensate for differences in ovary mass between the samples, we normalized each measured $\mathrm{T}$ and E2 concentration by the mass of the corresponding ovary sample. To standardize T and E2 concentrations for a typical fathead minnow ovary with a mean mass of $157.3 \mathrm{mg}$ (reference ovary mass), each normalized T and E2 concentration was multiplied by the reference ovary mass.

To estimate the mean ovary volume $(160.45 \mu \mathrm{L})$ for the reference ovary mass, we divided the ovary mass by the mean ovary density $\left(1.02 \mathrm{mg} \mu \mathrm{L}^{-1}\right)$. For a known ovary mass, the ovary density was determined from the mass of water displaced above the 5-mL mark of a 5 - $\mathrm{mL}$ graduated cylinder and dividing by the density of water (data not shown).

To compensate for differences in basal $\mathrm{T}$ and $\mathrm{E} 2$ concentrations between the baseline study and controls from the FAD study, we normalized the data. Each T concentration in the FAD study was scaled by the ratio of the mean $\mathrm{T}$ concentration at $14.5 \mathrm{~h}$ in the baseline study to the mean $\mathrm{T}$ concentration from the controls in the FAD study. The same normalization procedure was performed for E2.
Computational Steroidogenesis Model Overview

The computational model is based on an in vitro steroidogenesis experimental design with two compartments: culture medium and ovary tissue (Fig. 1). The model consists of transport and metabolic pathways. The transport pathways include ovary uptake of cholesterol (steroid precursor) and FAD, and secretion of newly synthesized androstenedione (AD), estrone (E1), T, and E2. The metabolic pathway includes conversion of cholesterol into $\mathrm{T}$ and $\mathrm{E} 2$, and inhibition of steroidogenic enzymes by FAD. Development of various aspects of the model is described in detail below.

\section{Ovary Uptake of Cholesterol}

Cholesterol is the precursor for all steroid hormones. The delivery of cholesterol to the inner mitochondrial membrane (site of the first metabolic reaction for steroid synthesis) consists of two main transport steps. First, exogenous cholesterol is imported into the cell mainly from low-density lipoprotein (LDL) via the LDL receptor-mediated lysosomal pathway. ${ }^{7,11,14}$ Second, cholesterol is transferred to the inner mitochondrial membrane by the intracellular sterol carrier protein-2, steroidogenic acute regulatory protein, and peripheral benzodiazepine receptor. ${ }^{11,14,18}$ We model the rate of cholesterol uptake from the medium as a zero-order process (Fig. 2) since the large

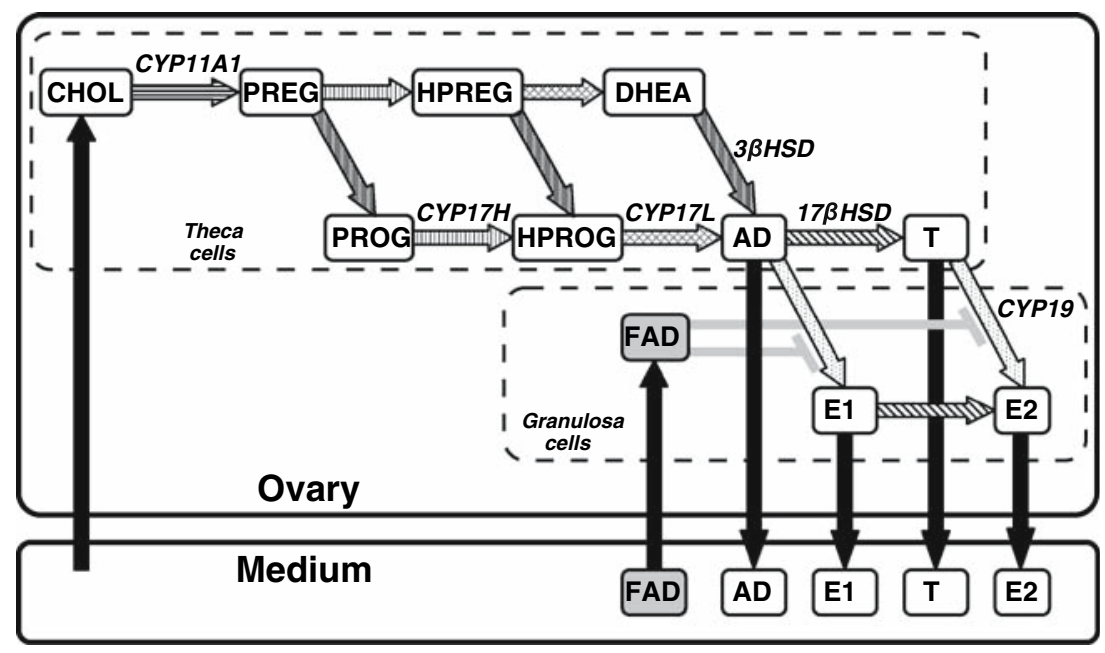

FIGURE 1. Conceptual steroidogenesis model for in vitro baseline and fadrozole-exposed ovaries. The model consists of two compartments, medium and ovary tissue. Transport processes, which occur between the medium and ovary are depicted by black arrows. Irreversible metabolic reactions, which occur in the ovary are depicted by arrows with each pattern representing a unique enzyme. Six enzymes labeled in italic next to reactions they catalyze are: cytochrome P450scc (side-chain-cleavage) (CYP11A1), cytochrome P450c17 ahydroxylase (CYP17H), cytochrome P450c17,20-lyase (CYP17L), 3- $\beta$-hydroxy-dehydrogenase (3 $\beta$ HSD), 17- $\beta$ hydroxy-dehydrogenase (17 $\beta \mathrm{HSD})$, and cytochrome P450 aromatase (CYP19). Steroids and their precursors are: cholesterol (CHOL), pregnenolone (PREG), 17 $\alpha$-hydroxypregnenolone (HPREG), dehydroepiandrosterone (DHEA), progesterone (PROG), $17 \alpha$-hydroxyprogesterone (HPROG), androstenedione (AD), estrone (E1), testosterone (T), and estradiol (E2). The endocrine active compound, FAD, is shown as an inhibitor of CYP19. 


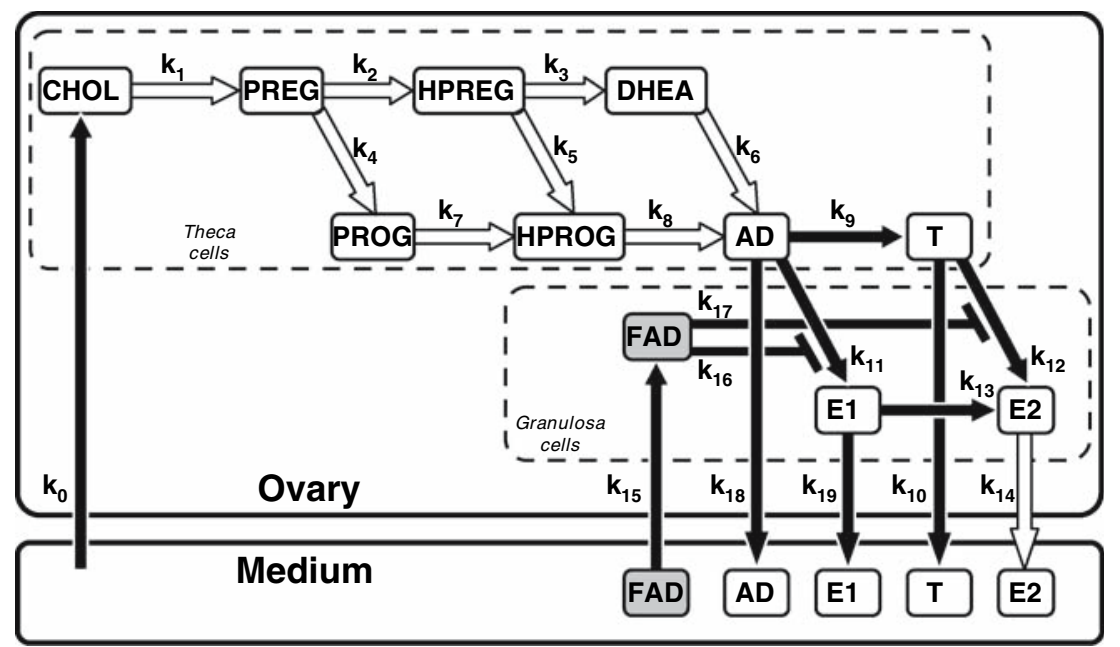

FIGURE 2. Graphical representation of the parameters for the mathematical model of ovarian steroidogenesis. Zero-order rate constant for cholesterol uptake into the ovary is $k_{0}$. Partition coefficient for FAD is $k_{15}$. First-order rate constants are: $k_{10}, k_{14}, k_{15}$, $k_{18}, k_{19}$ for transport processes; and $k_{1}-k_{9}, k_{11}-k_{13}$ for metabolic processes. Inhibition constants of FAD are $k_{16}$ and $k_{17}$ for E1 and E2 pathways, respectively. For the steady-state model, T and E2 medium concentrations are independent of the processes shown as white arrows, and dependent with the processes shown as black arrows.

concentration of cholesterol added to the medium for our in vitro steroidogenesis assay is probably sufficient to saturate the cholesterol transport process for the duration of the experiments.

\section{Ovary Uptake and Enzyme Inhibition of FAD}

A variety of EACs can inhibit steroidogenic enzymes involved the metabolic pathway. In this study, we examined the $\mathrm{T}$ and $\mathrm{E} 2$ responses to in vitro ovary exposures to the EAC, FAD. FAD is a competitive inhibitor of cytochrome P450 aromatase (CYP19), which catalyzes two different reactions in the metabolic pathway: conversion of AD to E1, and conversion of T to E2 (Fig. 1). ${ }^{4,16,32,48}$ We assume FAD in the medium diffuses into the ovary and reaches equilibrium with the FAD concentration in the ovary:

$$
C_{\mathrm{FAD}, \text { ovy }}=k_{15} C_{\mathrm{FAD}, \text { med }}
$$

where $C_{\text {FAD,ovy }}$ and $C_{\mathrm{FAD} \text {,med }}$ are the ovary and medium FAD concentrations, respectively; and $k_{15}$ is the partition coefficient (Fig. 2). For the two CYP19 reactions competitively inhibited by FAD, the kinetic parameters $k_{11}$ and $k_{12}$ are respectively divided by $\alpha_{\mathrm{E} 1}=1+\left(C_{\mathrm{FAD}, \text { ovy }} / k_{16}\right)$ and $\alpha_{\mathrm{E} 2}=1+\left(C_{\mathrm{FAD}, \text { ovy }} /\right.$ $\left.k_{17}\right)$ with FAD inhibition constants, $k_{16}$ and $k_{17}$ (Fig. 2, Appendix B).

\section{Metabolic Reactions}

The metabolic pathway in the ovary that converts cholesterol into $\mathrm{T}$ and $\mathrm{E} 2$ consists of 10 metabolites and 12 enzymatic reactions catalyzed by six different proteins (Fig. 1). ${ }^{27}$ In the ovary, the steroidogenic metabolic pathway spans two cell types; theca cells and granulosa cells. In theca cells, nine reactions in the pathway convert cholesterol into AD and T. In granulosa cells, the remaining three enzymatic reactions in the pathway convert $\mathrm{AD}$ and $\mathrm{T}$ into E1 and E2. All reactions occur in the smooth endoplasmic reticulum, except conversion of cholesterol into pregnenolone, which occurs in the inner mitochondrial membrane. ${ }^{1,21}$ Interorganelle and intercellular transports are not included in the model since these processes are assumed to be not rate-limiting and experimental data for these transport rates are unavailable. In addition, since all of the reactions are predominantly irreversible, ${ }^{13}$ we set the rates for the reverse reactions to zero. For each reaction, we assume the substrate concentration is much less than the Michaelis constant (substrate concentration that results in a half-maximal reaction rate). Hence, the rate of product formation increases linearly with substrate concentration as described by a first-order rate constant (Fig. 2).

\section{Secretion of Steroids}

The secretion of newly formed AD, T, E1, and E2 from the ovary to the medium is mediated by nonvesicular and vesicular transport. ${ }^{11,18,26}$ As described above, the steroidogenic metabolic pathway spans two cell types; theca cells and granulosa cells (Fig. 1). In theca cells, cholesterol is converted to AD and T. In granulosa cells, AD and T are converted to E1 and E2. In vitro, $\mathrm{AD}$ can be released from theca cells with subsequent uptake by the peripheral granulosa cells to form $\mathrm{E} 1$, or secreted into the medium. In vivo, $\mathrm{AD}$ released from theca cells can enter the capillaries for 
possible conversion to $\mathrm{T}$ or $\mathrm{E} 1$ in other tissues. ${ }^{8,9,16}$ Similarly, E1, a weak estrogen hormone, can be released from granulosa cells with subsequent secretion into the medium, in vitro, or plasma, in vivo, to be stored in other tissues for possible conversion to the more active estrogen, E2. ${ }^{8,16,31} \mathrm{We}$ assume the rate of secretion is a first-order process (Fig. 2).

\section{Dynamic Mass Balances}

The concentration dynamics of substrates are described by dynamic mass balances. We can express the dynamic mass balance for the steroids in the ovary volume $V_{\text {ovy }}$ and medium volume $V_{\text {med }}$, respectively, as:

$$
\begin{gathered}
V_{\text {ovy }} \frac{d C_{x, \text { ovy }}}{d t}=P_{x, \text { ovy }}-U_{x, \text { ovy }}+I_{x, \text { ovy }}-S_{x, \text { ovy }} \\
V_{\text {med }} \frac{d C_{x, \text { med }}}{d t}=S_{x, \text { ovy }}
\end{gathered}
$$

where $C_{x, \text { ovy }}$ and $C_{x, \text { med }}$ is the concentration of substrate $x$ in ovary and medium, respectively, $P_{x, \text { ovy }}$ is the production rate of substrate $x$ in ovary, $U_{x, \text { ovy }}$ is the utilization rate of substrate $x$ in ovary, $I_{x, \text { ovy }}$ is the import rate of substrate $x$ into ovary, and $S_{x, \text { ovy }}$ is the secretion rate of substrate $x$ from ovary. The first two terms in the right side of Eq. (2) represent the net metabolic reaction rate of substrate $x$. The last two terms represent the net uptake or release rate of substrate $x$ in the ovary.

For substrates that exist only in the ovary, the right side of Eq. (2) contains just the net metabolic reaction term. Appendix B provides all the dynamic mass balance equations in the ovary and medium compartments.

\section{Steady-State Analysis}

We assume the steroidogenic pathway is operating at steady-state in baseline and FAD-exposed fish state, even though some of the steady-state concentrations may be different than normal. ${ }^{46}$ Second, the medium was supplemented with IBMX (3-isobutyl-1methylxanthene), a non-selective phosphodiesterase inhibitor, to help maintain cyclic AMP levels in the ovary to sustain transport of cholesterol from the cytosol to the inner mitochondrial membrane, the site that initiates the metabolic pathway, and to sustain other cyclic AMP dependent processes in the steroidogenesis pathway. Third, the initial concentration of cholesterol in the medium is expected to be sufficient to sustain steroid synthesis for the duration of the experiments. Fourth, the initial concentrations of $\mathrm{T}$ and E2 including their intermediate metabolites in the excised fish ovaries are expected to be approximately basal in vivo levels. We also followed a carefully designed protocol for the ovary explant assays, as described above, to maintain the integrity of the metabolites, proteins, and other essential cellular constituents, and the viability of the steroidogenic cells for the duration of the experiment. In addition, a linear regression line closely fits the time-course baseline data for the accumulation of $\mathrm{E} 2$ and $\mathrm{T}$ in the medium (Fig. 3). For the FAD study, we assume the steroidogenesis pathway achieves steady-state after a short transient time as the FAD diffuses into the ovary and reaches equilibrium.

To examine steady-state behavior, we set the differential equations to zero to yield algebraic equations for each steroid and precursor in the ovary tissue. Using the symbolic mathematical software Maple 10 (Maplesoft, Waterloo, Ontario, Canada), we determined the analytical solutions for medium concentrations of $\mathrm{T}, C_{\mathrm{T} \text {,med, }}$, and $\mathrm{E} 2, C_{\mathrm{E} 2 \text {,med, }}$, in terms of the parameters as:

$$
\begin{aligned}
& C_{\mathrm{T}, \text { med }}(t) \\
& =\frac{k_{0} k_{9} k_{10}\left(k_{16}+k_{15} C_{\mathrm{FAD}, \text { med }}\right)\left(k_{17}+k_{15} C_{\mathrm{FAD}, \mathrm{med}}\right) t}{D_{1} D_{2}}
\end{aligned}
$$

$$
\begin{aligned}
C_{\mathrm{E} 2, \text { med }}(t)= & \frac{k_{0}\left(k_{13} k_{11} k_{16} k_{10} k_{17}+k_{13} k_{11} k_{16} k_{10} k_{15} C_{\mathrm{FAD}, \text { med }}+k_{13} k_{11} k_{16} k_{12} k_{17}\right) t}{D_{1} D_{2}\left(k_{19}+k_{13}\right)} \\
& +\frac{k_{0}\left(k_{12} k_{17} k_{9} k_{16} k_{19}+k_{12} k_{17} k_{9} k_{16} k_{13}+k_{12} k_{17} k_{9} k_{15} k_{19} C_{\mathrm{FAD}, \text { med }}+k_{12} k_{17} k_{9} k_{13} k_{15} C_{\mathrm{FAD}, \text { med }}\right) t}{D_{1} D_{2}\left(k_{19}+k_{13}\right)}
\end{aligned}
$$

ovaries during our in vitro experiments. There is good evidence to support this assumption. First, most metabolic systems operate close to a steady state, in which all inputs and outputs are in balance. ${ }^{46}$ Even in a disease state, a metabolic system is typically in steady- where $\quad D_{1}=k_{9} k_{16}+k_{9} k_{15} C_{\mathrm{FAD}, \text { med }}+k_{11} k_{16}+$ $k_{18} k_{16}+k_{18} k_{15} C_{\mathrm{FAD}, \mathrm{med}}$, and $D_{2}=k_{10} k_{17}+$ $k_{10} k_{15} C_{\mathrm{FAD} \text {,med }}+k_{12} k_{17}$. The equations show that $C_{\mathrm{T} \text {,med }}$ and $C_{\mathrm{E} 2 \text {,med }}$ depend on 11 parameters (black arrows in Fig. 2), which include five transport 


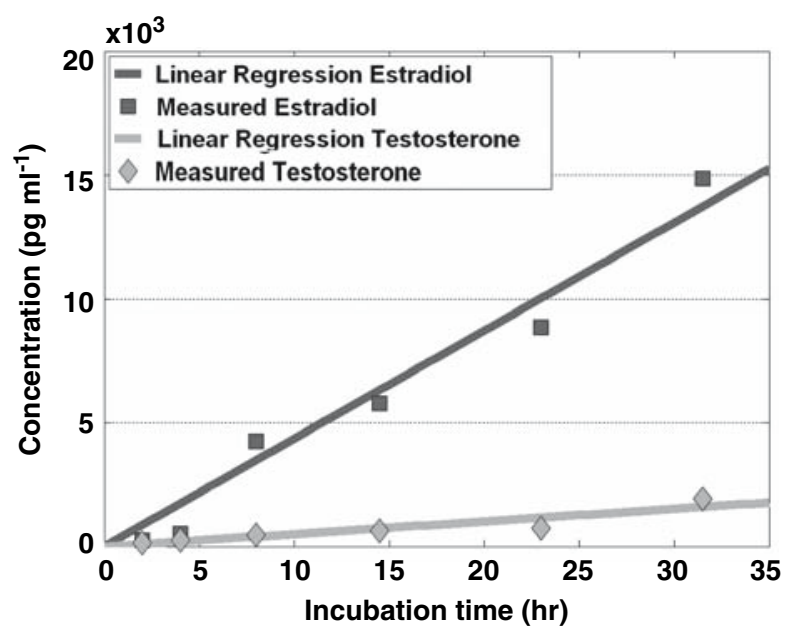

FIGURE 3. Comparison of linear regression line with timecourse data from baseline experiments. Linear regression lines for T and E2 were plotted as a function of time, and compared with mean concentrations measured at six time points between 2.0 and $31.5 \mathrm{~h}$. Mean E2 concentrations were much higher than $\mathrm{T}$ concentrations.

parameters $\left(k_{0}, k_{10}, k_{15}, k_{18}, k_{19}\right)$, four enzyme rate constants $\left(k_{9}, k_{11}, k_{12}, k_{13}\right)$, and two enzyme inhibition constants $\left(k_{16}, k_{17}\right)$. These equations for $C_{\mathrm{T}, \mathrm{med}}$ and $C_{\mathrm{E} 2 \text {,med }}$, and the 11 parameters are used in all subsequent analysis.

\section{Parameter Estimation}

The model was simultaneously fit to the mean concentration from replicate experiments in the baseline study and the FAD-exposure study. Parameters $k_{11}$ and $k_{12}$ were set to reported literature values from fish experiments (Table 1), ${ }^{49}$ and the remaining parameters were estimated with data from the baseline and FAD studies using the least squares method, and let $C_{\mathrm{T} \text {,med }}\left(t_{i} ; C_{\mathrm{FAD} \text {,med }}^{d}, \vec{k}\right)$ and $C_{\mathrm{E} 2 \text {,med }}\left(t_{i} ; C_{\mathrm{FAD} \text {,med }}^{d}, \vec{k}\right)$

TABLE 1. Estimated parameters from in vitro ovary data and those reported in literature ${ }^{a}$.

\begin{tabular}{lll}
\hline Parameter & \multicolumn{1}{c}{ Value } & \multicolumn{1}{c}{ Units } \\
\hline$k_{0}$ & 15401.470 & $\mathrm{pg} \mathrm{mL}^{-1} \mathrm{~h}^{-1}$ \\
$k_{9}$ & 0.509 & $\mathrm{~h}^{-1}$ \\
$k_{10}$ & 1726.553 & $\mathrm{~h}^{-1}$ \\
$k_{11}$ & $5.8(49)$ & $\mathrm{h}^{-1}$ \\
$k_{12}$ & $3.2(49)$ & $\mathrm{h}^{-1}$ \\
$k_{13}$ & 356.217 & $\mathrm{~h}^{-1}$ \\
$k_{15}$ & 0.0015 & $\mathrm{Dimensionless}$ \\
$k_{16}$ & 8143.017 & $\mathrm{pg} \mathrm{mL}^{-1}$ \\
$k_{17}$ & 4671.198 & $\mathrm{pg} \mathrm{mL}^{-1}$ \\
$k_{18}$ & 149.301 & $\mathrm{~h}^{-1}$ \\
$k_{19}$ & 102.171 & $\mathrm{~h}^{-1}$ \\
\hline
\end{tabular}

${ }^{a}$ References for values from literature are given in parenthesis.. be the model-predicted concentrations of $\mathrm{T}$ and $\mathrm{E} 2$ for the $d$ th FAD dose (including control) $C_{\text {FAD,med }}^{d}$ at the $i$ th time $t_{i}$ with parameter set $\vec{k}$, respectively. Let $C_{\mathrm{T}, \text { med }}^{d, i}$ and $C_{\mathrm{E} 2 \text {,med }}^{d, i}$ be the measured concentration of $\mathrm{T}$ and $\mathrm{E} 2$ for the $d$ th FAD dose (including control) $C_{\text {FAD,med }}^{d}$ at the $i$ th time $t_{i}$, respectively, where $\vec{k}=\left(k_{0}, k_{9}, k_{10}, k_{11}, k_{12}, k_{13}, k_{15}, k_{16}, k_{17}, k_{18}, k_{19}\right)$, $d=1, \ldots, 6$, and $i=1, \ldots, n_{d}$. Then, the least squares estimate, $\vec{k}^{*}=\left(k_{0}^{*}, k_{9}^{*}, k_{10}^{*}, k_{11}^{*}, k_{12}^{*}, k_{13}^{*}, k_{15}^{*}, k_{16}^{*}, k_{17}^{*}, k_{18}^{*}\right.$, $k_{19}^{*}$ ), is the parameter values $\vec{k}$, which minimizes the cost function

$$
\begin{aligned}
J(\vec{k})= & \sum_{d=1}^{6} \sum_{i=1}^{n_{d}}\left(C_{\mathrm{T}, \text { med }}^{d, i}-C_{\mathrm{T}, \text { med }}\left(t_{i} ; C_{\mathrm{FAD}, \text { med }}^{d}, \vec{k}\right)\right)^{2} \\
& +\left(C_{\mathrm{E} 2, \text { med }}^{d, i}-C_{\mathrm{E} 2 \text {,med }}\left(t_{i} ; C_{\mathrm{FAD}, \text { med }}^{d}, \vec{k}\right)\right)^{2}
\end{aligned}
$$

Parameters were estimated with an iterative optimization algorithm using MATLAB 7.2 (Mathworks, Natick, MA, USA) software. We chose the NelderMead simplex method for its relative insensitivity to the initial parameter values as compared to other popular methods, such as Newton's method, and its robustness to discontinuities. ${ }^{25}$ Convergence to the solution was confirmed after the parameter search terminated.

\section{Sensitivity Analysis}

To examine model uncertainty, we performed a sensitivity analysis on the 11 parameters. To rank the sensitivities of the parameters, we calculated relative sensitivities, $R S T_{k_{i}}$ and $R S E 2_{k_{i}}$, with respect to parameter $k_{i}$ for the model-predicted concentrations $C_{\mathrm{T}, \text { med }}$ and $C_{\mathrm{E} 2 \text {,med }}$, respectively:

$$
\begin{aligned}
R S T_{k_{i}}\left(C_{\mathrm{T}, \mathrm{med}}, k_{i}\right) & =\frac{\delta C_{\mathrm{T}, \mathrm{med}}}{\delta k_{i}}\left(\frac{k_{i}}{C_{\mathrm{T}, \mathrm{med}}}\right) \\
R S E 2_{k_{i}}\left(C_{\mathrm{E} 2, \mathrm{med}}, k_{i}\right) & =\frac{\delta C_{\mathrm{E} 2 \text { med }}}{\delta k_{i}}\left(\frac{k_{i}}{C_{\mathrm{E} 2, \text { med }}}\right)
\end{aligned}
$$

Using Maple, partial derivatives were analytically determined for $C_{\mathrm{T} \text {,med }}$ and $C_{\mathrm{E} 2 \text {,med }}$ with respect to parameter $k_{i}$, and expressions for relative sensitivity functions were obtained. For control and each FAD dose, we evaluated the relative sensitivity functions. Magnitudes of relative sensitivities indicate the degree to which changes in parameter values lead to changes in model outputs, negative values indicate an inverse relationship between a parameter change and the resulting model output change, and positive values indicate a direct relationship. 


\section{RESULTS}

The equations for $C_{\mathrm{T}, \mathrm{med}}$ and $C_{\mathrm{E} 2 \text {,med }}$ expressed in terms of the model parameters showed that 9 out of 20 total parameters initially considered in the steady-state model were eliminated; $\mathrm{T}$ and $\mathrm{E} 2$ are dependent on only 11 parameters (Eqs. 4 and 5). As shown by the graphical representation of the model (Fig. 2), T and E2 are independent of processes upstream of AD except for the cholesterol uptake rate $\left(k_{0}\right)$, and dependent with processes downstream of AD except for the E2 secretion rate $\left(k_{14}\right)$.

The estimated parameters for the steroidogenesis model are shown in Table 1. The time for convergence to the solution for the iterative parameter estimation was typically $5 \mathrm{~s}$ on a Pentium $\mathrm{M}$ class computer using MATLAB.

The dynamic medium concentrations of $\mathrm{T}$ and $\mathrm{E} 2$ measured in the baseline study are shown in Fig. 3. From multiple independent experiments, the minimum detection limits of the RIA method used to quantify E2 and $\mathrm{T}$ are $21.5 \pm 8.9 \mathrm{pg} \mathrm{mL}^{-1}$ (mean $\pm \mathrm{SD}$ ) with $N=12$ and $17.2 \pm 8.7 \mathrm{pg} \mathrm{mL}^{-1}$ with $N=14$, respectively. The coefficient of variations of the combined extraction and RIA technical variability for E2 and T are $9.9 \%$ with $N=8$ and $10.3 \%$ with $N=13$, respectively. A linear regression line corresponds well to the mean time-course data with $R^{2}$ values of 0.94 and 0.98 for T and E2, respectively. Since the medium accumulates $\mathrm{T}$ and $\mathrm{E} 2$, the close fit of a linear regression line to the T and E2 time-course data indicates steroid synthesis and secretion in the in vitro fish ovary is operating near steady-state.

We compared the model-predicted concentrations of $\mathrm{T}$ and $\mathrm{E} 2$ to the time-course data from baseline experiments. The model-predicted $\mathrm{T}$ and $\mathrm{E} 2$ concentrations correspond well to the mean time-course data (Fig. 4), which closely match a linear regression line (Fig. 3).

Model-predicted dose-response behavior was compared with mean medium concentrations of $\mathrm{T}$ and E2 measured after a $14.5 \mathrm{~h}$ FAD exposure at five different doses and a control (Fig. 5). For E2, the model-predicted concentrations closely correspond to the mean measurements that decrease as FAD increases, except at the lowest FAD concentration $(6.497 \times$ $10^{5} \mathrm{pg} \mathrm{mL}^{-1}$ ) where the mean E2 data is greater than control. The mean E2 measurements for FAD concentrations at $2.165 \times 10^{7} \mathrm{pg} \mathrm{mL}^{-1}$ and $6.497 \times$ $10^{7} \mathrm{pg} \mathrm{mL}^{-1}$ were statistically different from control $(p<0.0001)$. For T, the model-predicted concentration compare well with the mean data that remained approximately unchanged as FAD increases. The measured $\mathrm{T}$ concentrations for FAD exposures were not statistically different from controls.

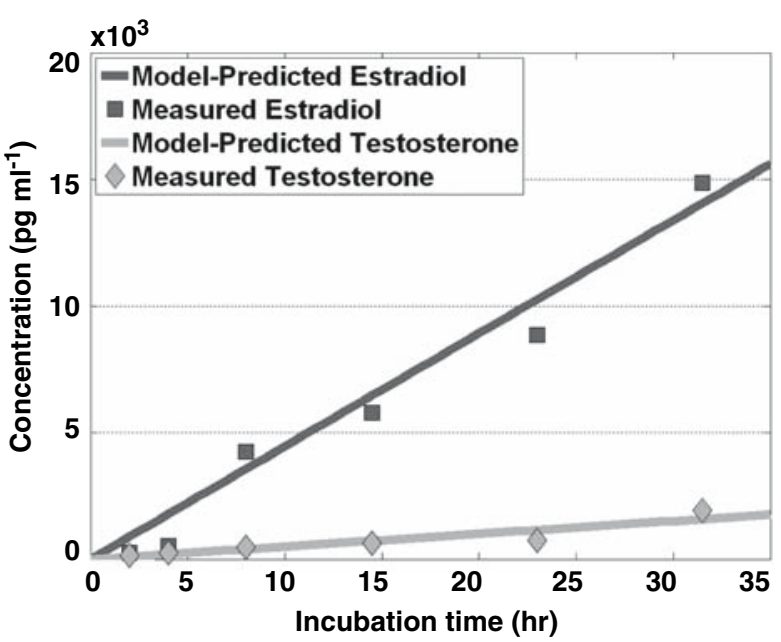

FIGURE 4. Comparison of model-predictions with timecourse data from baseline experiments. Model-predicted concentrations of T and E2 in the medium were plotted as a function of time, and compared with mean concentrations measured at six points. The same data are shown in Fig. 3.

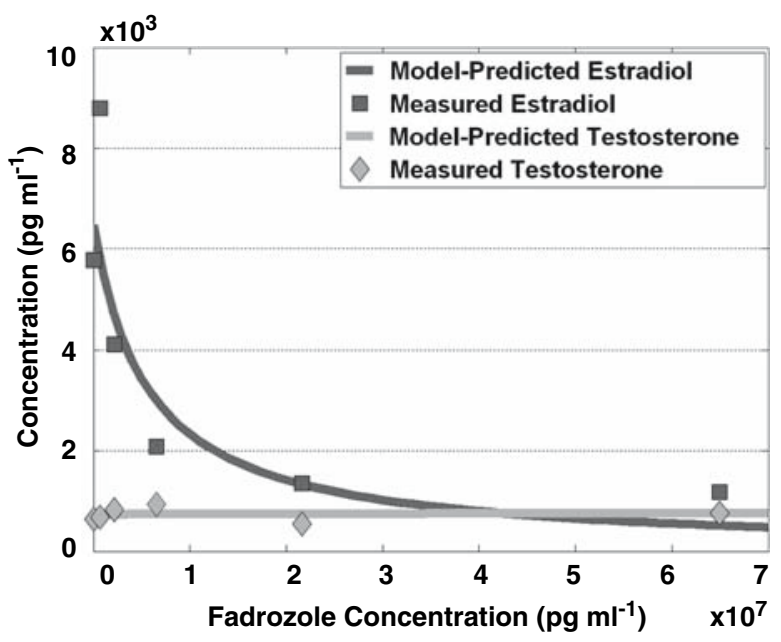

FIGURE 5. Comparison of model-predicted and dose-response data after $14.5 \mathrm{~h}$ incubation of ovary explants with FAD. Model-predicted T and E2 concentrations in the medium were plotted as a function of FAD concentration, and compared with mean concentrations measured for one control and five FAD concentrations.

The relative sensitivities of $\mathrm{T}$ and $\mathrm{E} 2$ are shown in Fig. 6. At every dose, four parameters were highly sensitive: $k_{0}$ and $k_{18}$ for both $\mathrm{T}$ and $\mathrm{E} 2, k_{9}$ for $\mathrm{T}$, and $k_{11}$ for E2. For E2, two parameters $k_{13}$ and $k_{19}$ were moderately sensitive at every dose, and two parameters $k_{15}$ and $k_{16}$ had dose-dependent sensitivity; their sensitivity increased as FAD increased. The E1 pathway appears to be the preferred pathway for E2 synthesis since E2 was sensitive to the E1 pathway and its inhibition $\left(k_{11}, k_{13}, k_{16}\right)$, and not sensitive to the $\mathrm{T}$ pathway and its inhibition $\left(k_{9}, k_{12}, k_{17}\right)$. The sensitivity 

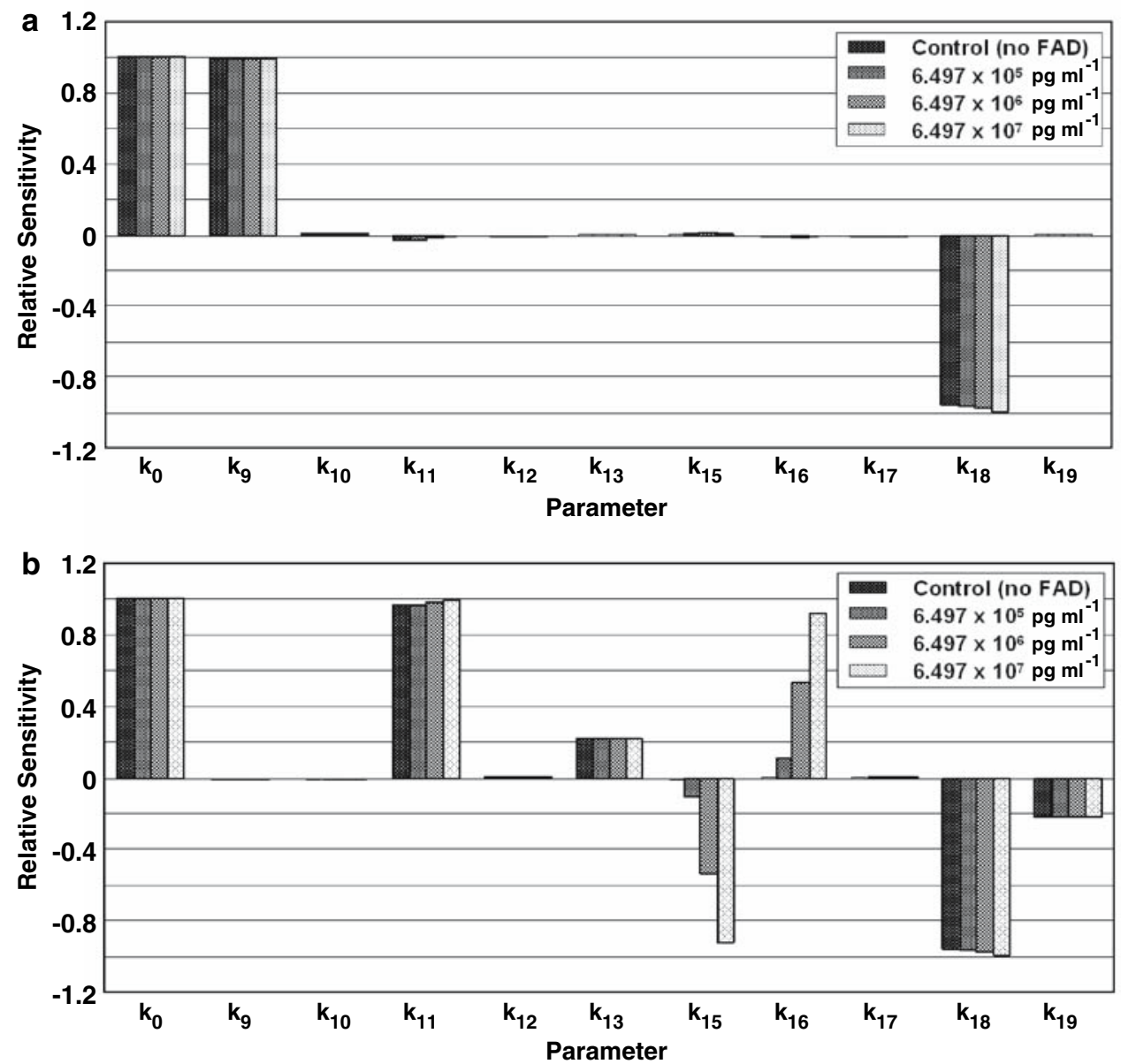

FIGURE 6. Relative sensitivities for the model outputs, T (a) and E2 (b), are plotted as a function of the 11 model parameters ( $k_{0}$, $k_{9}-k_{13}, k_{15}-k_{19}$ ) for a control (no FAD) and the lowest, middle, and highest FAD concentrations of $6.497 \times 10^{5}, 6.497 \times 10^{6}$, and $6.497 \times 10^{7} \mathrm{pg} \mathrm{mL}^{-1}$, respectively. The relative sensitivities range from -1.0 to 1.0 ; negative values indicate an inverse relationship between a parameter change and the resulting model output change; positive values indicate a direct relationship. The magnitudes of relative sensitivities indicate the degree to which changes in parameter values lead to changes in model outputs; percentage change of model output for a given percentage change of parameter.

of $\mathrm{T}$ and $\mathrm{E} 2$ can indicate the uncertainty of the parameters. The parameters with high sensitivity tend to have less uncertainty as compared to parameters with low sensitivity.

\section{DISCUSSION}

Our goal was to develop a mechanistic mathematical model of the intraovarian transport and metabolic processes to predict the synthesis and secretion of $T$ and E2, and their responses to FAD. The model-predicted medium concentrations of $\mathrm{T}$ and $\mathrm{E} 2$ closely correspond to the time-course data from baseline (control) experiments, and dose-response data from experiments with FAD-exposed fathead minnow ovarian preparations. The steady-state model reduced the complexity of the model while maintaining the model's predictive ability.
The potential importance of this model arises from its use of mechanistic information at the biochemical level to predict dose-response behaviors. Usually, laboratory experiments are performed at high doses due to the minimum detection levels of the assays, while humans are typically exposed to environmental and pharmaceutical chemicals at significantly lower doses. Extrapolations in dose-response curves from high dose data may be inaccurate if not guided by mechanistic information. $^{12}$

To enable prediction of the dose-response for EACexposed ovaries, our steroidogenesis model included each enzymatic reaction in the metabolic pathway. Under baseline physiologic conditions, the rate-limiting step is the transport of cholesterol from outer to inner mitochondrial membrane. ${ }^{11,22}$ For EAC-exposed ovaries, one or more steps in the pathway could become rate-limiting, depending on the EAC concentration and enzyme inhibition constant. Some compounds 
inhibit a single specific steroidogenic enzyme, while other compounds inhibit multiple enzymes. ${ }^{3,17}$ In addition, a sensitivity analysis indicated the E1 pathway as the preferred pathway for E2 synthesis, as compared to the $\mathrm{T}$ pathway for control and FAD-exposed ovaries. Our model and analysis methods provide a framework for the investigation of these behaviors and should, after further development, allow for more accurate predictions of dose-response for EAC effects on steroid production. In addition, the model will facilitate study of the effects of mixtures of EAC with different mechanisms of action.

As shown with the in vitro data, explant ovaries can provide the data needed for comparison with model predictions. Explant ovary experiments eliminate the feedback of the hypothalamus-pituitary-gonadal axis, and allow for steroid precursors, to be added to medium to isolate regions of the pathway. For example, additions of AD to ovary explants would isolate the segment of the steroidogenesis pathway downstream of AD. In addition, explant ovary experiments with various EAC at measured tissue exposure concentrations could be useful to refine the model structure and parameter estimates by perturbing or selectively blocking certain reactions within the steroidogenesis pathway.

This study demonstrated the feasibility of a mechanistic steroidogenesis model for predicting levels of $\mathrm{T}$ and E2 produced and secreted by ovarian preparations. Adjustments of the model for vertebrates other than fish, including mammals, would only require different sets of parameter values, since the qualitative structure of the model would not change.

FAD has a large effect on E2 concentrations, and little or no effect on $\mathrm{T}$ concentrations in the medium (Fig. 5). There is good evidence that this response is due to enzyme inhibition of CYP19 and not experimental error. FAD is a reversible, competitive inhibitor of CYP19 (Fig. 1). ${ }^{33,35}$ It has been reported to inhibit other enzymes but only at concentrations 4-5 orders of magnitude greater than those needed to inhibit CYP19, which are higher concentrations than those used in this study. ${ }^{6,30}$ In our study, the sensitivity analysis shows that E2 is sensitive to the uptake and inhibition of FAD $\left(k_{15}, k_{16}\right)$, while T is not sensitive to either the uptake or inhibition of $\operatorname{FAD}\left(k_{15}, k_{16}, k_{17}\right)$. Moreover, the measured $\mathrm{T}$ concentrations for FAD exposures were not statistically different than controls. In addition, the minimum detection limits of the RIA method used to quantify E2 and T are similar with means of 21.5 and $17.2 \mathrm{pg} \mathrm{mL}^{-1}$, respectively. The combined extraction and RIA technical variability for $\mathrm{E} 2$ and $\mathrm{T}$ are also similar with coefficient of variations of 9.9 and $10.3 \%$, respectively.

Although our model predictions compare well with the experimental data, the model-predicted medium concentrations of E2 do not correspond at one data point; the mean data at the lowest FAD concentration $\left(6.497 \times 10^{5} \mathrm{pg} \mathrm{mL}^{-1}\right)$. Instead of a decrease in E2 production as predicted by the model, the ovary increased E2 production relative to controls. A possible source of this discrepancy is the assumption of constant enzyme concentrations. FAD can induce the production of the enzyme, CYP19, most likely to compensate for the enzyme's inhibition by FAD. ${ }^{44}$ At low FAD doses, CYP19 increases may overcompensate for the inhibition by FAD and increase $\mathrm{E} 2$ production relative to controls. At higher FAD doses, CYP19 levels may saturate and E2 production decreases relative to controls. If this hypothesis is supported in future experiments at low FAD doses with measurements of transcripts and proteins levels, modifications to the model should incorporate the dose-dependent induction of CYP19 production by FAD.

\section{ACKNOWLEDGMENTS}

We thank Dr. Haluk Özkaynak, Dr. Jerry Blancato, and Dr. Robert Kavlock for their assistance and helpful suggestions, and Lindsay Blake and Katie Greene for their assistance with the in vitro experiments. This study was funded in part through the National Center for Computational Toxicology of the U.S. Environmental Protection Agency (US EPA) Office of Research and Development (ORD). Miyuki Breen was supported by an NSF-VIGRE Fellowship: NSF-DMS0354189-EMSW21-VIGRE Project: VIGRE-II - "Integrated and Mentored Program of Research and Education in Statistical Sciences." This manuscript was reviewed by the US EPA and approved for publication but does not necessarily reflect Agency policy. Mention of products or trade names does not indicate endorsement by the federal government. Conclusions drawn in this study neither constitute nor necessarily reflect US EPA policy regarding the test chemicals, test methods, and models. Laboratory procedures involving animals were conducted in accordance with protocols reviewed and approved by the US EPA Mid-Continent Ecology Division's Animal Care and Used Committee in compliance with Animal Welfare Act regulations and Interagency Research Animal Committee guidelines.

\section{APPENDIX A: KINETIC EQUATIONS FOR THE METABOLIC REACTIONS}

The enzymatic reactions are modeled as first-order processes. Rate constants, $k_{11}$ and $k_{12}$, were calculated from literature reported values of the Michaelis 
constant, $K_{\mathrm{m}}$, and catalysis rate, $k_{\mathrm{cat}}$, in goldfish ovaries $^{49}$ and our measured microsomal protein content, $P_{\text {ovy }}$ and density, $D_{\text {ovy }}$, in fathead minnow ovaries (data not shown) as:

$$
k_{11}=\frac{V_{\max }}{K_{\mathrm{m}}}
$$

where $V_{\text {max }}=k_{\text {cat }} P_{\text {ovy }} D_{\text {ovy }}, P_{\text {ovy }}=3.7 \mathrm{mg}$ microsomal protein/g ovary, and $D_{\text {ovy }}=1.02 \mathrm{mg} \mu \mathrm{L}^{-1}$. For $k_{12}$, Eq. (A1) was used, but with different literature reported values for $K_{\mathrm{m}}$ and $k_{\mathrm{cat}}$.

\section{APPENDIX B: DYNAMIC MASS BALANCE EQUATIONS IN OVARY AND MEDIUM}

1. CHOL in ovary:

$V_{\text {ovy }} \frac{d C_{\mathrm{CHOL}, \text { ovy }}(t)}{d t}=V_{\text {med }} k_{0}-k_{1} V_{\text {ovy }} C_{\mathrm{CHOL}, \text { ovy }}(t)$

2. PREG in ovary:

$$
\begin{aligned}
\frac{d C_{\text {PREG,ovy }}(t)}{d t}= & k_{1} C_{\mathrm{CHOL}, \text { ovy }}(t) \\
& -\left(k_{2}+k_{4}\right) C_{\mathrm{PREG}, \text { ovy }}(t)
\end{aligned}
$$

3. HPREG in ovary:

$$
\begin{aligned}
\frac{d C_{\text {HPREG }, \text { ovy }}(t)}{d t}= & k_{2} C_{\text {PREG }, o v y}(t) \\
& -\left(k_{3}+k_{5}\right) C_{\text {HPREG }, \text { ovy }}(t)
\end{aligned}
$$

4. DHEA in ovary:

$\frac{d C_{\text {DHEA ,ovy }}(t)}{d t}=k_{3} C_{\text {HPREG,ovy }}(t)-k_{6} C_{\text {DHEA,ovy }}(t)$

5. PROG in ovary:

$$
\frac{d C_{\text {PROG }, o v y}(t)}{d t}=k_{4} C_{\text {PREG }, o v y}(t)-k_{7} C_{\text {PROG }, \text { ovy }}(t)
$$

6. HPROG in ovary:

$$
\begin{aligned}
\frac{d C_{\mathrm{HPROG}, \text { ovy }}(t)}{d t}= & k_{5} C_{\text {HPREG,ovy }}(t)+k_{7} C_{\mathrm{PROG}, \text { ovy }}(t) \\
& -k_{8} C_{\mathrm{HPROG}, \text { ovy }}(t)
\end{aligned}
$$

7. AD in ovary:

$$
\begin{aligned}
\frac{d C_{\mathrm{AD}, \text { ovy }}(t)}{d t}= & k_{6} C_{\mathrm{DHEA}, \text { ovy }}(t)+k_{8} C_{\mathrm{HPROG}, \text { ovy }}(t) \\
& -\left(k_{9}+k_{18}+\frac{k_{11}}{\alpha_{E 1}}\right) C_{\mathrm{AD}, \text { ovy }}(t)
\end{aligned}
$$

8. T in ovary:

$$
\frac{d C_{\mathrm{T}, \mathrm{ovy}}(t)}{d t}=k_{9} C_{\mathrm{AD}, \mathrm{ovy}}(t)-\left(k_{10}+\frac{k_{12}}{\alpha_{\mathrm{E} 2}}\right) C_{\mathrm{T}, \mathrm{ovy}}(t)
$$

9. E1 in ovary:

$$
\frac{d C_{\mathrm{E} 1, \mathrm{ovy}}(t)}{d t}=\frac{k_{11}}{\alpha_{\mathrm{E} 1}} C_{\mathrm{AD}, \text { ovy }}(t)-\left(k_{13}+k_{19}\right) C_{\mathrm{E} 1, \mathrm{ovy}}(t)
$$

10. E2 in ovary:

$$
\begin{aligned}
\frac{d C_{\mathrm{E} 2, \text { ovy }}(t)}{d t}= & k_{13} C_{\mathrm{E} 1, \text { ovy }}(t)+\frac{k_{12}}{\alpha_{E 2}} C_{\mathrm{T}, \text { ovy }}(t) \\
& -k_{14} C_{\mathrm{E} 2, \text { ovy }}(t)
\end{aligned}
$$

11. AD in medium:

$$
\begin{aligned}
V_{\text {med }} \frac{d C_{\mathrm{AD}, \text { med }}(t)}{d t} & =k_{18} V_{\text {ovy }} C_{\mathrm{AD}, \text { ovy }}(t) ; \\
C_{\mathrm{AD}, \text { med }}(0) & =0
\end{aligned}
$$

12. E1 in medium:

$$
\begin{aligned}
V_{\text {med }} \frac{d C_{\mathrm{E} 1, \text { med }}(t)}{d t} & =k_{19} V_{\text {ovy }} C_{\mathrm{E} 1, \text { ovy }}(t) ; \\
C_{\mathrm{E} 1, \text { med }}(0) & =0
\end{aligned}
$$

13. $\mathrm{T}$ in medium:

$$
V_{\text {med }} \frac{d C_{\mathrm{T}, \text { med }}(t)}{d t}=k_{10} V_{\text {ovy }} C_{\mathrm{T}, \text { ovy }}(t) ; \quad C_{\mathrm{T}, \text { med }}(0)=0
$$

14. E2 in medium:

$$
\begin{aligned}
V_{\text {med }} \frac{d C_{\mathrm{E} 2, \text { med }}(t)}{d t} & =k_{14} V_{\text {ovy }} C_{\mathrm{E} 2, \text { ovy }}(t) ; \\
C_{\mathrm{E} 2, \text { med }}(0) & =0
\end{aligned}
$$

\section{REFERENCES}

${ }^{1}$ Agarwal, A. K., and R. J. Auchus. Minireview: cellular redox state regulates hydroxysteroid dehydrogenase activity and intracellular hormone potency. Endocrinology 146:2531-2538, 2005.

${ }^{2}$ Ankley, G. T., K. M. Jensen, M. D. Kahl, J. J. Korte, and E. A. Makynen. Description and evaluation of a short-term reproduction test with the fathead minnow (Pimephales promelas). Environ. Toxicol. Chem. 20:1276-1290, 2001.

${ }^{3}$ Ankley, G. T., K. M. Jensen, M. D. Kahl, E. A. Makyen, L. S. Blake, K. J. Greene, and D. L. Villeneuve. Ketoconazole in the fathead minnow (pimephales promelas) reproductive toxicity and biological compensation. Environ. Toxicol. Chem. (in press).

${ }^{4}$ Ankley, G. T., M. D. Kahl, K. M. Jensen, M. W. Hornung, J. J. Korte, E. A. Makynen, and R. L. Leino. Evaluation of the aromatase inhibitor fadrozole in a short-term reproduction assay with the fathead minnow (Pimephales promelas). Toxicol. Sci. 67:121-130, 2002.

${ }^{5}$ Becker, S., C. Chubb, and L. Ewing. Mathematical model of steroidogenesis in rat and rabbit testes. Am. J. Physiol. 239:R184-R195, 1980 . 
${ }^{6}$ Bhatnagar, A. S., A. Hausler, K. Schieweck, M. Lang, and R. Bowman. Highly selective inhibition of estrogen biosynthesis by CGS 20267, a new non-steroidal aromatase inhibitor. J. Steroid Biochem. Mol. Biol. 37:1021-1027, 1990.

${ }^{7}$ Brown, M. S., and J. L. Goldstein. A receptor-mediated pathway for cholesterol homeostasis. Science 232:34-47, 1986.

${ }^{8}$ Budzinski, H., M. H. Deviar, P. Labadie, and A. Togola. Analysis of hormonal steroids in fish plasma and bile by coupling solid-phase extraction to GC/MS. Anal. Bioanal. Chem. 386:1429-1439, 2006.

${ }^{9}$ Burger, H. G. Androgen production in women. Fertil. Steril. 77:S3-S5, 2002.

${ }^{10}$ Buzdar, A., and A. Howell. Advances in aromatase inhibition: clinical efficacy and tolerability in the treatment of breast cancer. Clin. Cancer Res. 7:2620-2635, 2001.

${ }^{11}$ Chang, T. Y., C. C. Chang, N. Ohgami, and Y. Yamauchi. Cholesterol sensing, trafficking, and esterification. Аnnu. Rev. Cell Dev. Biol. 22:129-157, 2006.

${ }^{12}$ Conolly, R. B., and W. K. Lutz. Nonmonotonic doseresponse relationships: mechanistic basis, kinetic modeling, and implications for risk assessment. Toxicol. Sci. 77:151157, 2004.

${ }^{13}$ Durocher, F., R. Sanchez, M. L. Ricketts, Y. Labrie, V. Laudet, and J. Simard. Characterization of the guinea pig 3beta-hydroxysteroid dehydrogenase/Delta5-Delta4-isomerase expressed in the adrenal gland and gonads. J. Steroid Biochem. Mol. Biol. 97:289-298, 2005.

${ }^{14}$ Gallegos, A. M., J. K. Schoer, O. Starodub, A. B. Kier, J. T. Billheimer, and F. Schroeder. A potential role for sterol carrier protein-2 in cholesterol transfer to mitochondria. Chem. Phys. Lipids 105:9-29, 2000.

${ }^{15}$ Jensen, K. M., J. J. Korte, M. D. Kahl, M. S. Pasha, and G. T. Ankley. Aspects of basic reproductive biology and endocrinology in the fathead minnow (Pimephales promelas). Comp. Biochem. Physiol. C Toxicol. Pharmacol. 128:127-141, 2001

${ }^{16}$ Kochak, G. M., S. Mangat, M. T. Mulagha, E. A. Entwistle, R. J. Santen, A. Lipton, and L. Demers. The pharmacodynamic inhibition of estrogen synthesis by fadrozole, an aromatase inhibitor, and its pharmacokinetic disposition. J. Clin. Endocrinol. Metab. 71:1349-1345, 1990.

${ }^{17}$ Laignelet, L., J. F. Narbonne, J. C. Lhuquenot, and J. L. Riviere. Induction and inhibition of rat liver cytochrome(s) P-450 by an imidazole fungicide (prochloraz). Toxicology 59:271-284, 1989.

${ }^{18}$ Maxfield, F. R., and D. Wustner. Intracellular cholesterol transport. J. Clin. Invest. 110:891-898, 2002.

${ }^{19}$ McMaster, M. E., K. R. Munkittrick, J. J. Jardine, R. D. Robinson, and G. J. Van Der Kraak. Protocol for measuring in vitro steroid production by fish gonadal tissue. Canadian Technical Report of Fisheries and Aquatic Sciences 1961. Department of Fisheries and Oceans, Burlington, Ontario, Canada, 1995.

${ }^{20}$ Miller, W. L. Molecular biology of steroid hormone synthesis. Endocr. Rev. 9:295-318, 1988.

${ }^{21}$ Miller, W. L. Minireview: regulation of steroidogenesis by electron transfer. Endocrinology 146:2544-2550, 2005.

${ }^{22}$ Miller, W. L., and J. F. Strauss 3rd. Molecular pathology and mechanism of action of the steroidogenic acute regulatory protein, StAR. J. Steroid Biochem. Mol. Biol. 69:131-141, 1999.

${ }^{23}$ Murphy, C. A., K. A. Rose, and P. Thomas. Modeling vitellogenesis in female fish exposed to environmental stressors: predicting the effects of endocrine disturbance due to exposure to a PCB mixture and cadmium. Reprod. Toxicol. 19:395-409, 2005.

${ }^{24}$ Nahoum, V., A. Gangloff, R. Shi, and S. X. Lin. How estrogen-specific proteins discriminate estrogens from androgens: a common steroid binding site architecture. Faseb. J. 17:1334-1336, 2003.

${ }^{25}$ Nelder, J. A., and R. Mead. A simplex method for function minimization. Comp. J. 7:308-313, 1965.

${ }^{26}$ Neufeld, E. B., A. M. Cooney, J. Pitha, E. A. Dawidowicz, N. K. Dwyer, P. G. Pentchev, and E. J. Blanchette-Mackie. Intracellular trafficking of cholesterol monitored with a cyclodextrin. J. Biol. Chem. 271:21604-21613, 1996.

${ }^{27}$ Payne, A. H., and D. B. Hales. Overview of steroidogenic enzymes in the pathway from cholesterol to active steroid hormones. Endocr. Rev. 25:947-970, 2004.

${ }^{28}$ Sanderson, J. T., J. Boerma, G. W. Lansbergen, and M. van den Berg. Induction and inhibition of aromatase (CYP19) activity by various classes of pesticides in H295R human adrenocortical carcinoma cells. Toxicol. Appl. Pharmacol. 182:44-54, 2002.

${ }^{29}$ Sanderson, J. T., R. J. Letcher, M. Heneweer, J. P. Giesy, and M. van den Berg. Effects of chloro-s-triazine herbicides and metabolites on aromatase activity in various human cell lines and on vitellogenin production in male carp hepatocytes. Environ. Health Perspect. 109:1027-1031, 2001.

${ }^{30}$ Santen, R. J., P. Langecker, S. J. Santner, S. Sikka, J. Rajfer, and R. Swerdloff. Potency and specificity of CGS 16949A as an aromatase inhibitor. Endocr. Res. 16:77-91, 1990.

${ }^{31}$ Santen, R. J., L. M. Demers, J. Lynch, H. Harvey, A. Lipton, M. Mulagha, J. Hanagan, J. E. Garber, I. C. Henderson, and R. M. Navari, et al.. Specificity of low dose fadrozole hydrochloride (CGS 16949A) as an aromatase inhibitor. J. Clin. Endocrinol. Metab. 73:99-106, 1991.

${ }^{32}$ Santen, R. J., and H. A. Harvey. Use of aromatase inhibitors in breast carcinoma. Endocr. Relat. Cancer 6:7592, 1999.

${ }^{33}$ Schieweck, K., A. S. Bhatnagar, and A. Matter. CGS 16949A, a new nonsteroidal aromatase inhibitor: effects on hormone-dependent and-independent tumors in vivo. Cancer Res. 48:834-838, 1988.

${ }^{34}$ Selgrade, J. F., and P. M. Schlosser. A model for the production of ovarian hormones during the menstrual cycle. Fields Inst. Commun. 21:429-446, 1999.

${ }^{35}$ Steele, R. E., L. B. Mellor, W. K. Sawyer, J. M. Wasvary, and L. J. Browne. In vitro and in vivo studies demonstrating potent and selective estrogen inhibition with the nonsteroidal aromatase inhibitor CGS 16949A. Steroids 50:147-161, 1999.

${ }^{36}$ Stocco, D. M., and B. J. Clark. Regulation of the acute production of steroids in steroidogenic cells. Endocr. Rev. 17:221-244, 1996.

${ }^{37}$ U.S. Environmental Protection Agency. User's Guide for Conducting Life-cycle Chronic Toxicity Tests with Fathead Minnows (Pimephales promelas). Duluth, MN: EPA-600 881-011, 1982.

${ }^{38}$ U.S. Environmental Protection Agency. Guidelines for the Culture of Fathead Minnows Pimephales Promelas for Use in Toxicity Tests. Duluth, MN: EPA/600/3-87/001, 1987.

${ }^{39}$ U.S. Environmental Protection Agency. Pesticide Assessment Guidelines. Subdivision E, Hazard Evaluation: Wildlife and Aquatic Organisms. Washington, DC: EPA540 09-82 024, 1989. 
${ }^{40}$ U.S. Environmental Protection Agency. In: Methods for Measuring the Acute Toxicity of Effluents and Receiving Waters to Freshwater and Marine Organisms, 4th edn., edited by C. I. Weber. EPA-600 4-90 027. Office of Research and Development, Environmental Monitoring Systems Laboratory, Cincinnati, OH, 1991.

${ }^{41}$ U.S. Environmental Protection Agency. In: Short-Term Methods for Estimating the Chronic Toxicity of Effluents and Receiving Water to Freshwater Organisms, 3rd edn., edited by P. A. Lewis, D. J. Klemm, J. M. Lazorchak, T. J. Norberg-King, W. H. Peltier, and M. A. Heber. EPA 600 491 002. Office of Research and Development, Environmental Monitoring Systems Laboratory, Cincinnati, OH, 1994.

${ }^{42}$ U.S. Environmental Protection Agency. A short-term method for assessing the reproductive and developmental toxicity of endocrine-disrupting chemicals using the fathead minnow (Pimephales promelas). Duluth, MN: EPA600/R-01/067, 2002.

${ }^{43}$ Villeneuve, D. L., G. T. Ankley, E. A. Makynen, L. S. Blake, K. J. Greene, E. B. Higley, J. L. Newsted, J. P. Giesy, and M. Hecker. Comparison of fathead minnow ovary explant and H295R cell-based steroidogenesis assays for identifying endocrine active chemicals. Ecotox. Environ. Safe. (in press), doi:10.1016/j.ecoenv.2007.03.001.

${ }^{44}$ Villeneuve, D. L., I. Knoebl, M. D. Kahl, K. M. Jensen, D. E. Hammermeister, K. J. Greene, L. S. Blake, and G. T.
Ankley. Relationship between brain and ovary aromatase activity and isoform-specific aromatase mRNA expression in the fathead minnow (Pimephales promelas). Aqua. Toxicol. 76:353-368, 2006.

${ }^{45}$ Vinggaard, A. M., C. Hnida, V. Breinholt, and J. C. Larsen. Screening of selected pesticides for inhibition of CYP19 aromatase activity in vitro. Toxicol. In Vitro 14:227-234, 2000.

${ }^{46}$ Voit, E. O. Computational Analysis of Biochemical Systems: A Practical Guide for Biochemists and Molecular Biologists. Cambridge: Cambridge University Press, 2000.

${ }^{47}$ Walsh, L. P., C. N. Kuratko, and D. M. Stocco. Econazole and miconazole inhibit steroidogenesis and disrupt steroidogenic acute regulatory (StAR) protein expression posttranscriptionally. J. Steroid Biochem. Mol. Biol. 75:229236, 2000.

${ }^{48}$ Yue, W., and A. M. Brodie. Mechanisms of the actions of aromatase inhibitors 4-hydroxyandrostenedione, fadrozole, and aminoglutethimide on aromatase in JEG-3 cell culture. J. Steroid Biochem. Mol. Biol. 63:317-328, 1997.

${ }^{49}$ Zhao, J., P. Mak, A. Tchoudakova, G. Callard, and S. Chen. Different catalytic properties and inhibitor responses of the goldfish brain and ovary aromatase isozymes. Gen. Comp. Endocrinol. 123:180-191, 2001. 